Supporting Information for

\title{
Size effect in Hydrogenation of Nitroaromatics using Support-immobilized Atomically Precise Gold Clusters
}

\author{
Sam Nesbitt, *Matthew Watson and Vladimir B. Golovko*
}

Sam Nesbitt - Department of Chemical and Process Engineering, College of Engineering, University of Canterbury, Private Bag 4800, Christchurch 8140, New Zealand

and

The MacDiarmid Institute for Advanced Materials and Nanotechnology, School of Physical and Chemical Sciences, College of Science, University of Canterbury, Private Bag 4800, Christchurch 8140, New Zealand Matthew Watson - Department of Chemical and Process Engineering, College of Engineering, University of Canterbury, Private Bag 4800, Christchurch 8140, New Zealand Vladimir B. Golovko - The MacDiarmid Institute for Advanced Materials and Nanotechnology, School of Physical and Chemical Sciences, College of Science, University of Canterbury, Private Bag 4800, Christchurch 8140, New Zealand 


\section{Materials Characterization}

\subsection{NMR}

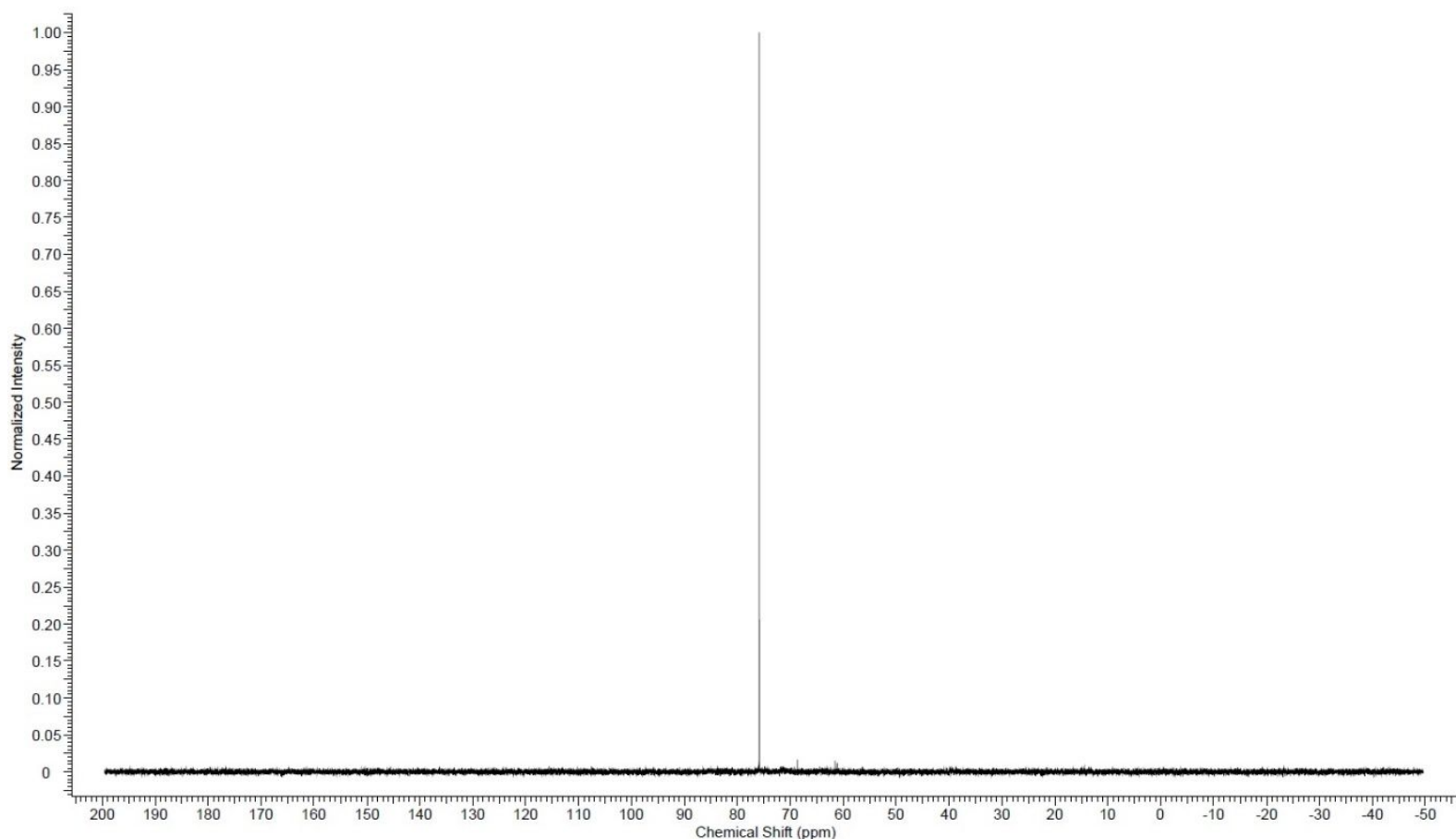

Figure S1. $\left[A u_{19}(P h C \equiv C)_{9}(H d p p a)_{3}\right]\left(S_{b F}\right){ }^{31} P$ NMR spectrum

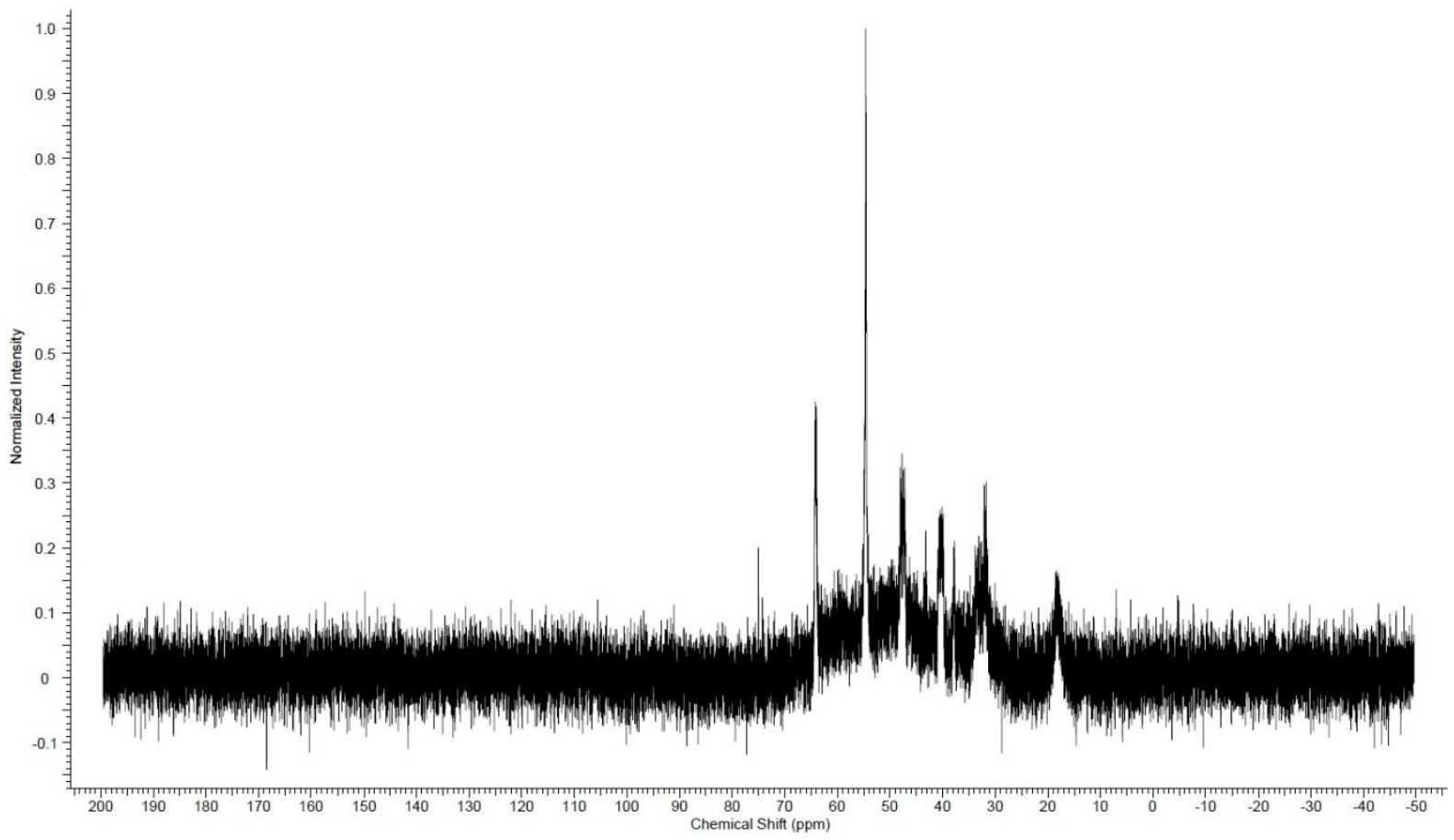

Figure $S 2$. $\left[\mathrm{Au}_{20}\left(\mathrm{PP}_{3}\right)_{4}\right](\mathrm{Cl})_{4}{ }^{31} \mathrm{P} \mathrm{NMR}$ spectrum 


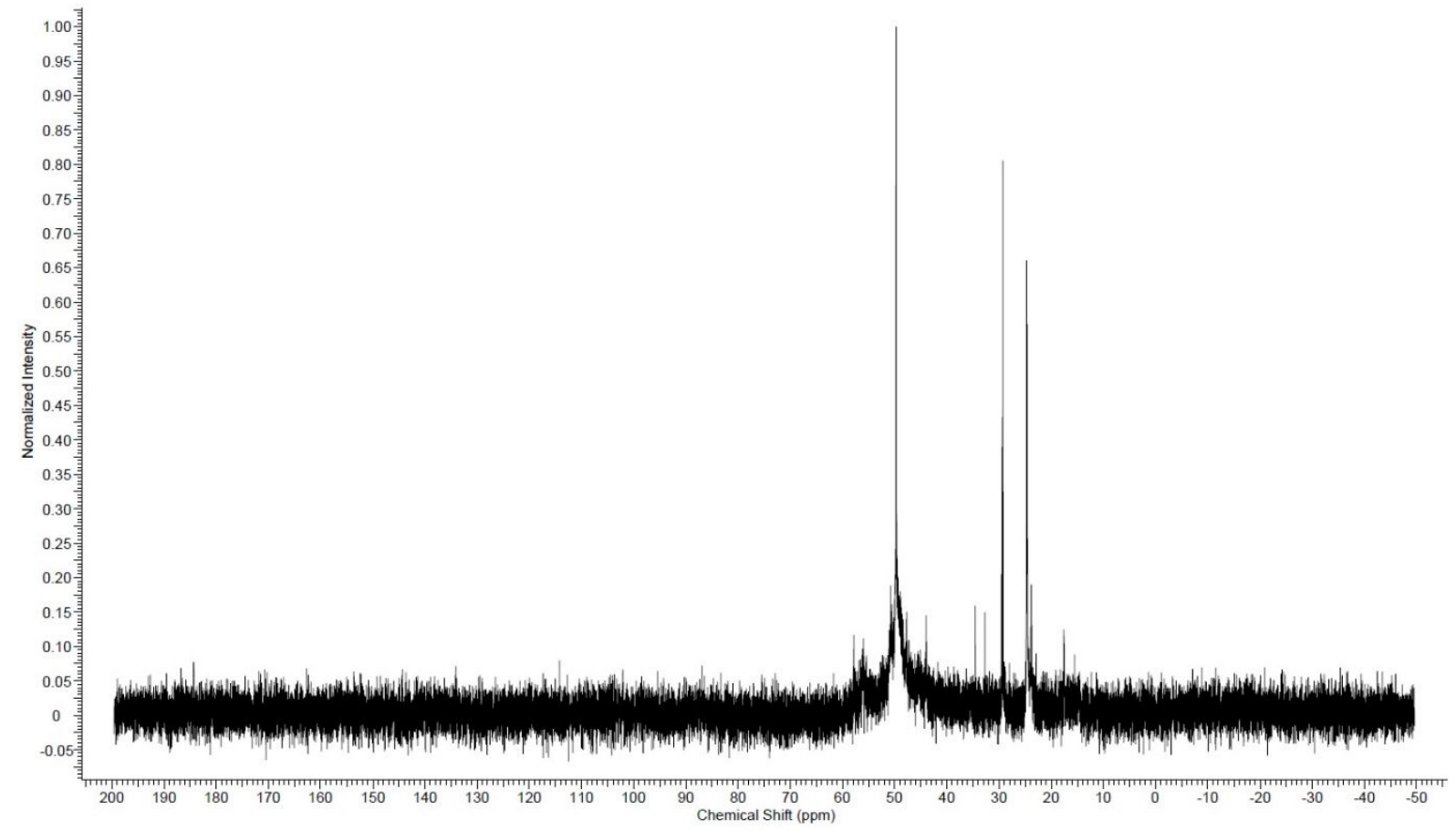

Figure S3. $\left[\mathrm{Au}_{22}(\text { Dppee })_{7}\right](\mathrm{Cl})_{2}{ }^{31} \mathrm{P}$ NMR spectrum

\subsection{Thermogravimetric Analysis}

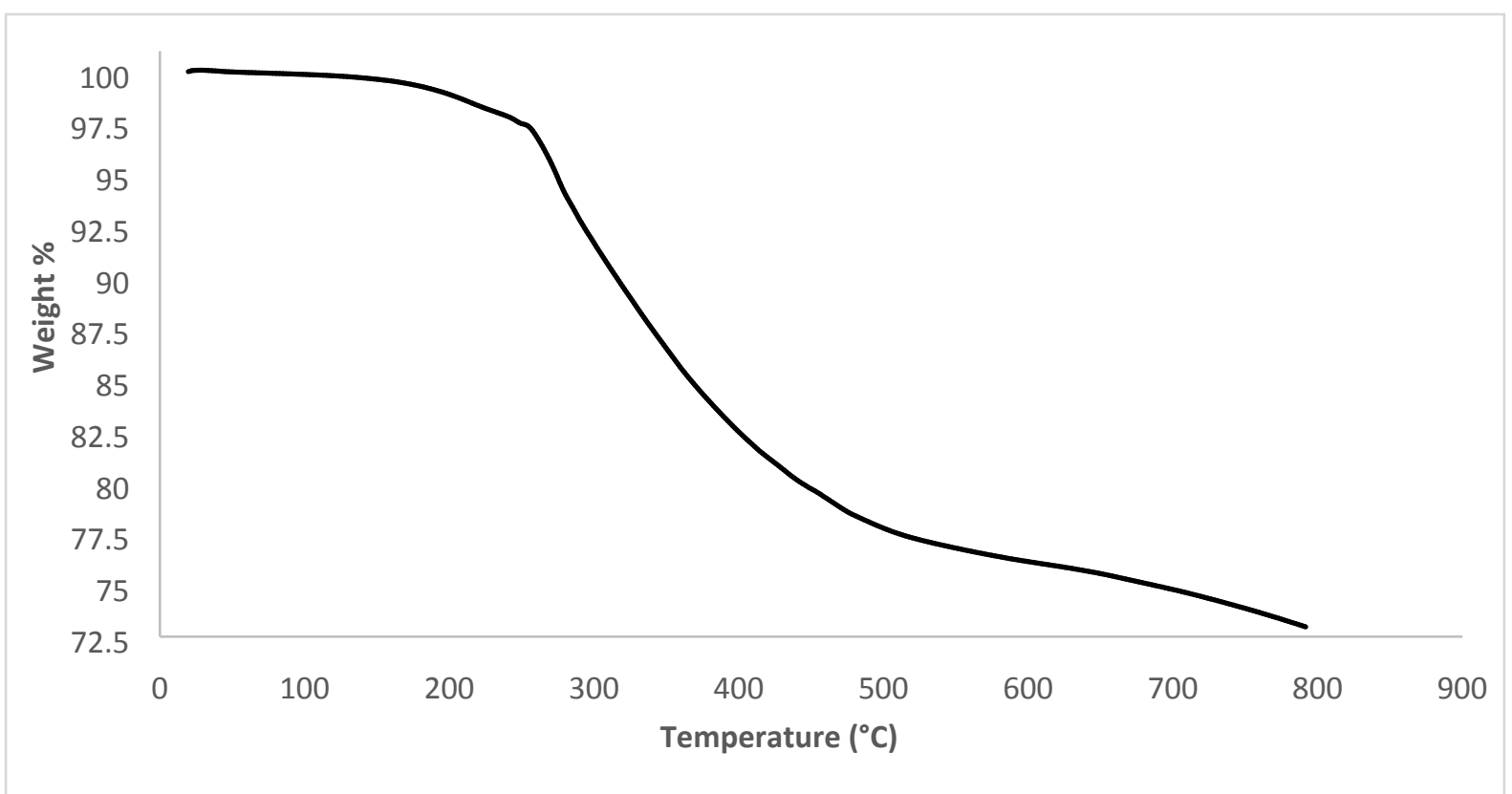

Figure S4. $\left[A u_{19}(P h C \equiv C)_{9}(H d p p a)_{3}\right]\left(S b F_{6}\right) T G A$ data 


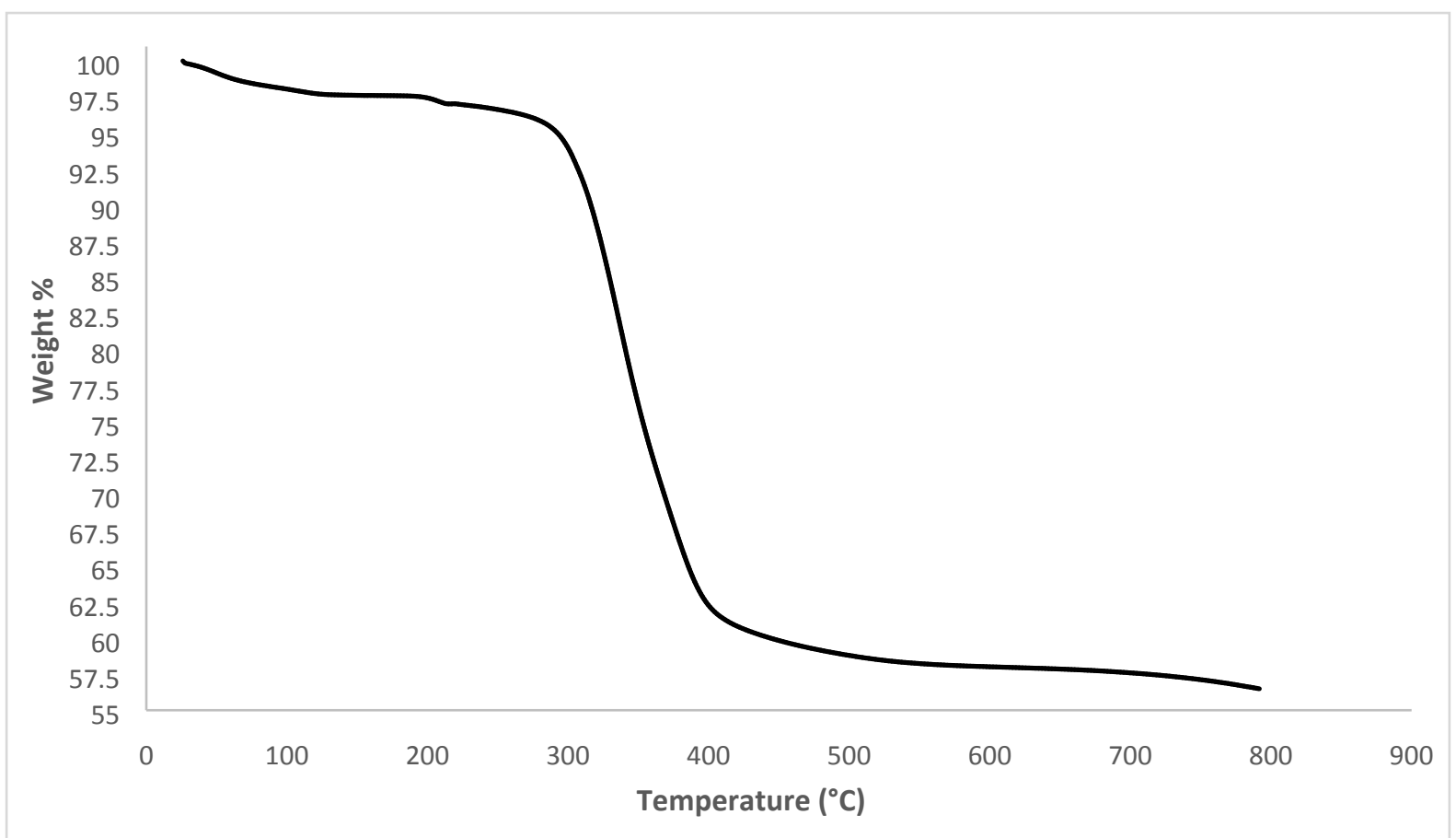

Figure S5. $\left[\mathrm{Au}_{20}\left(\mathrm{PP}_{3}\right)_{4}\right](\mathrm{Cl})_{4} \mathrm{TGA}$ data

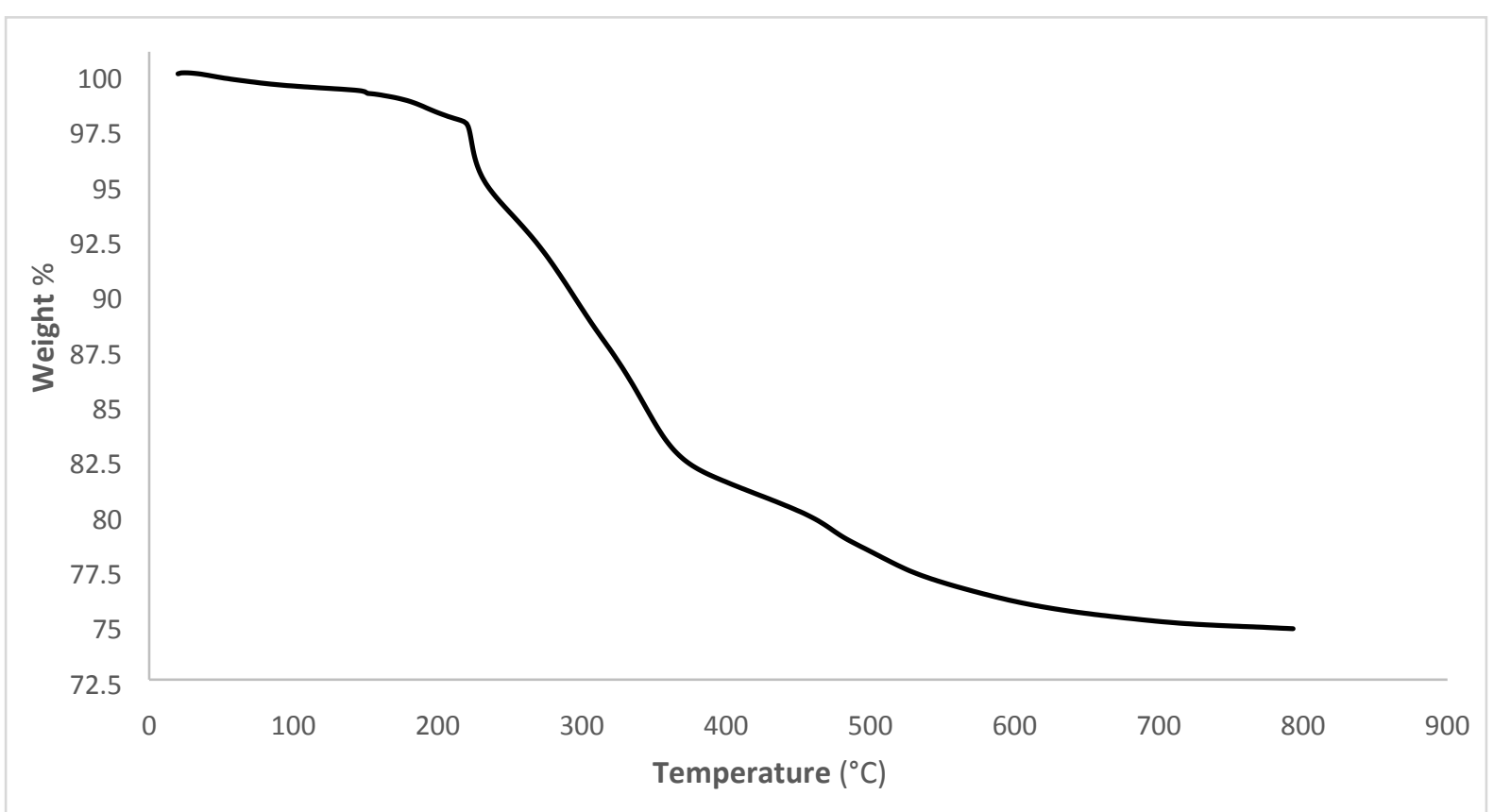

Figure S6. $\left[\mathrm{Au}_{22}(\text { Dppee })_{7}\right](\mathrm{Cl})_{2} \mathrm{TGA}$ data 


\subsection{UV-Vis spectra}

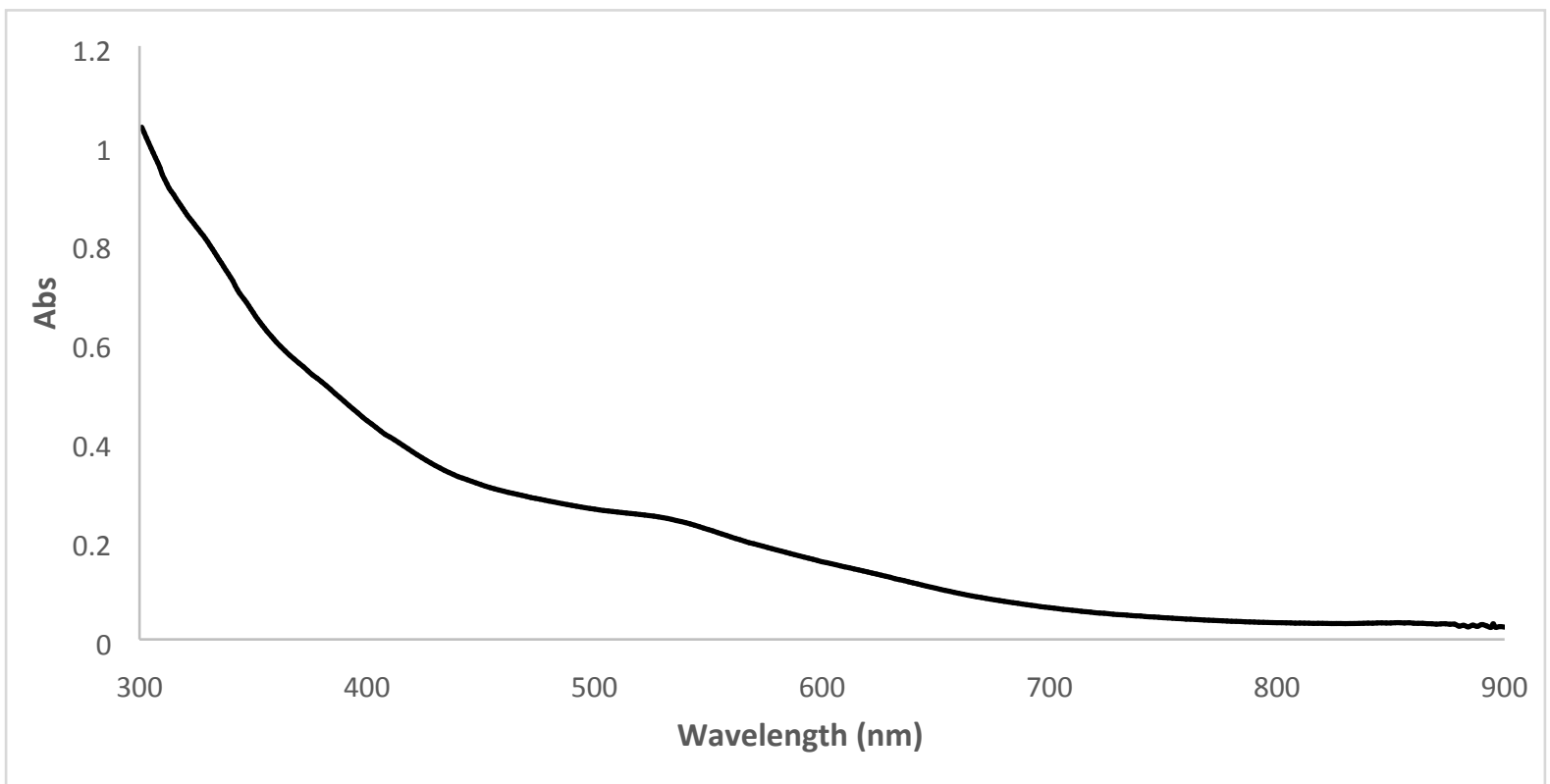

Figure S7. $\left[A u_{19}\left(\mathrm{PhC} \equiv \mathrm{C}_{9}(\mathrm{Hdppa})_{3}\right]\left(\mathrm{SbF}_{6}\right) \mathrm{UV}\right.$-Vis spectra

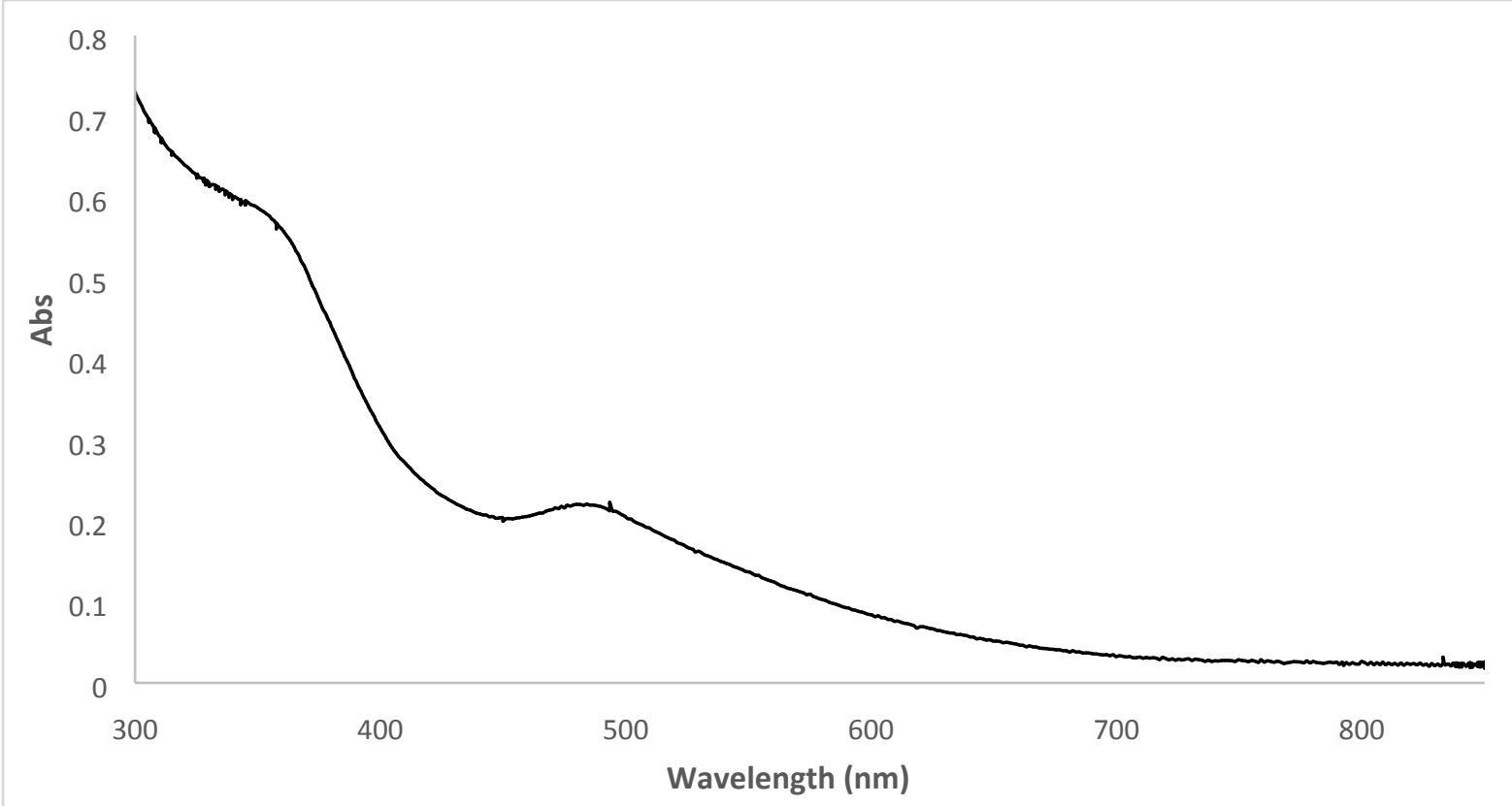

Figure S8. $\left[\mathrm{Au}_{20}\left(\mathrm{PP}_{3}\right)_{4}\right](\mathrm{Cl})_{4} U \mathrm{U}$-Vis spectra 


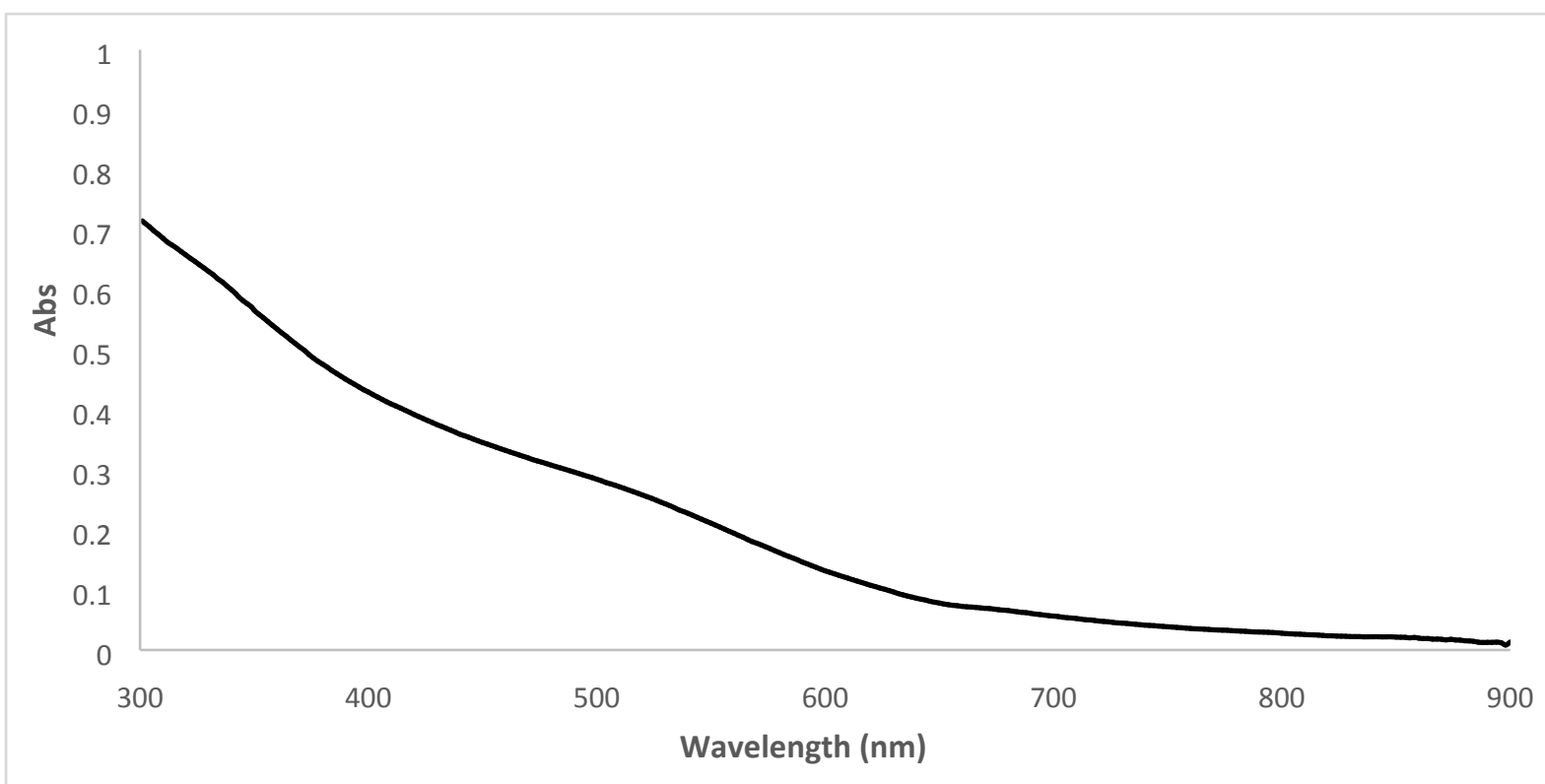

Figure S9. $\left[\mathrm{Au}_{22}(\text { Dppee })_{7}\right](\mathrm{Cl})_{2} \mathrm{UV}$-Vis spectra

\subsection{UV-Vis DR spectra of as made and activated materials}

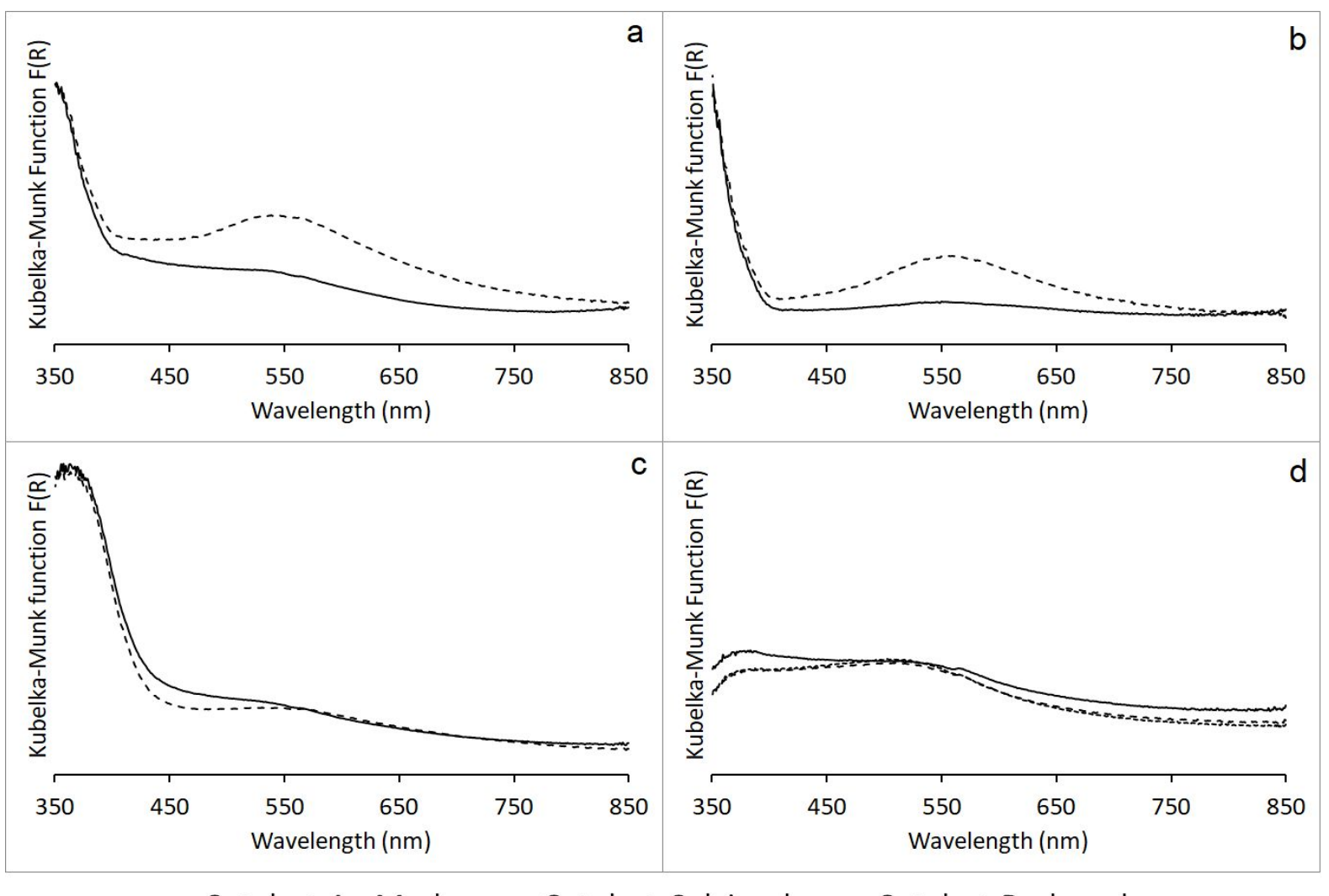

-Catalyst As-Made ----Catalyst Calcined -----Catalyst Reduced

Figure S10. UV-Vis DR Spectra of (a) 0.2\% Au $19 /$ Anatase, (b) $0.2 \%$ Au $19 /$ Degussa P25, (c) $0.2 \% \mathrm{Au}_{19} / \mathrm{CeO}_{2}$, (d) $0.2 \% \mathrm{Au}_{19} / \mathrm{HAP}$ 

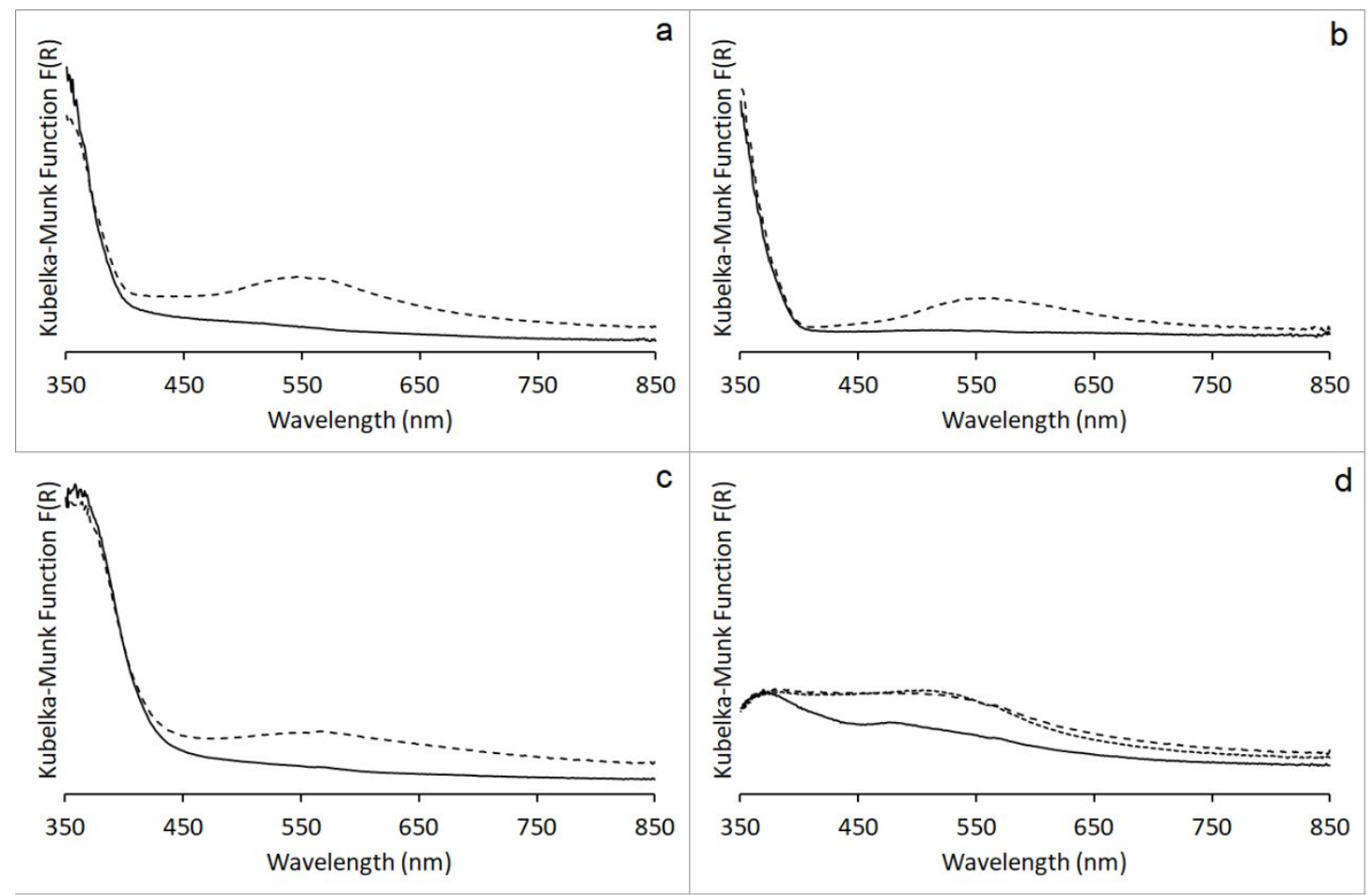

-Catalyst As-Made ----Catalyst Calcined -.--.-Catalyst Reduced

Figure S11. UV-VIS DRS Spectra of (a) $0.2 \%$ Au 20 Anatase, (b) $0.2 \%$ Au 20 Degussa P25, (c) $0.2 \% \mathrm{Au}_{20} / \mathrm{CeO}_{2}$, (d) $0.2 \% \mathrm{Au}_{20} / \mathrm{HAP}$

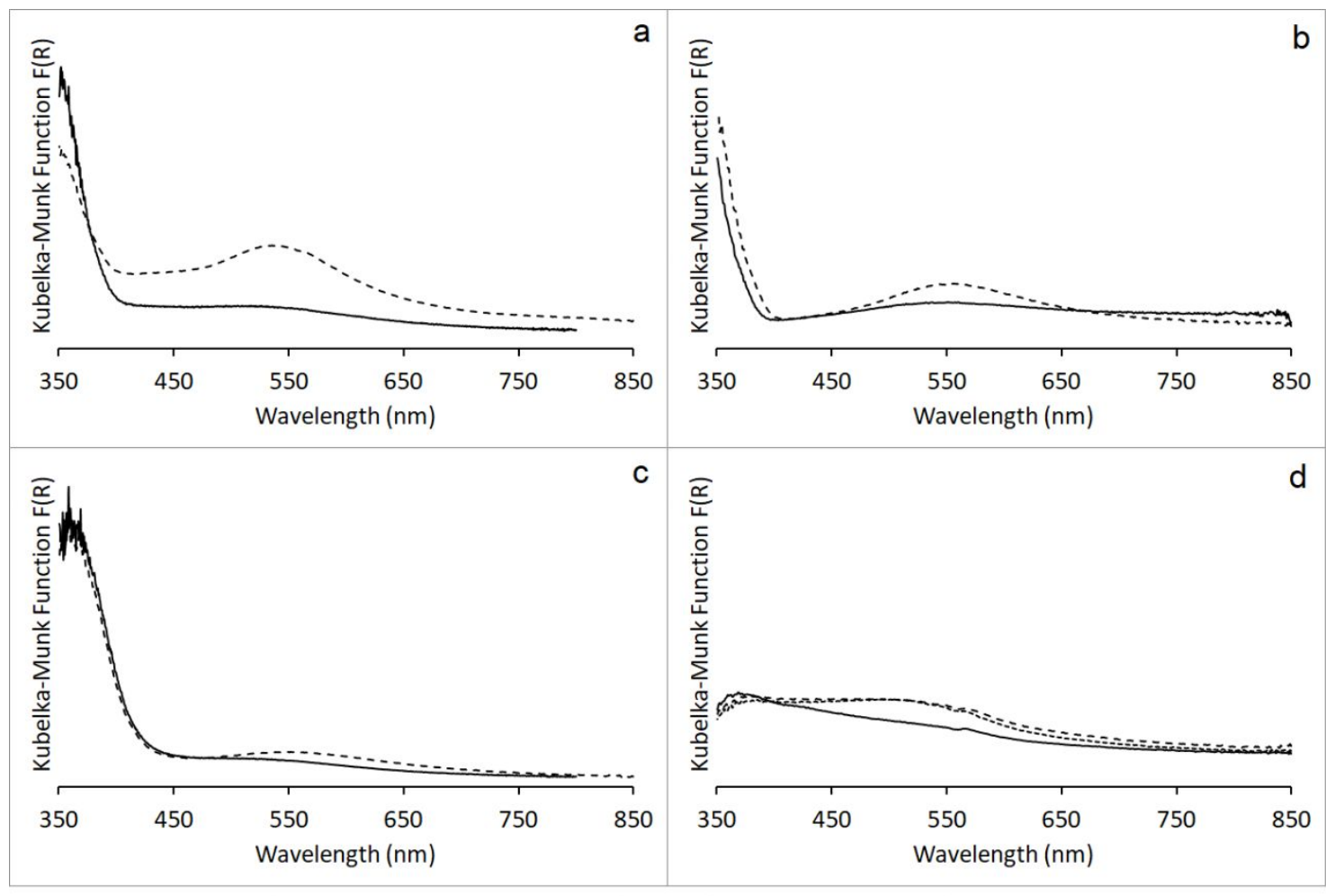

-Catalyst As-Made ---Catalyst Calcined -.-.-Catalyst Reduced

Figure S12. UV-VIS DRS Spectra of (a) $0.2 \%$ Au 22 /Anatase, (b) $0.2 \%$ Au 22 /Degussa P25, (c) $0.2 \% \mathrm{Au}_{22} / \mathrm{CeO}_{2}$, (d) $0.2 \% \mathrm{Au}_{22} / \mathrm{HAP}$ 


\subsection{PXRD}
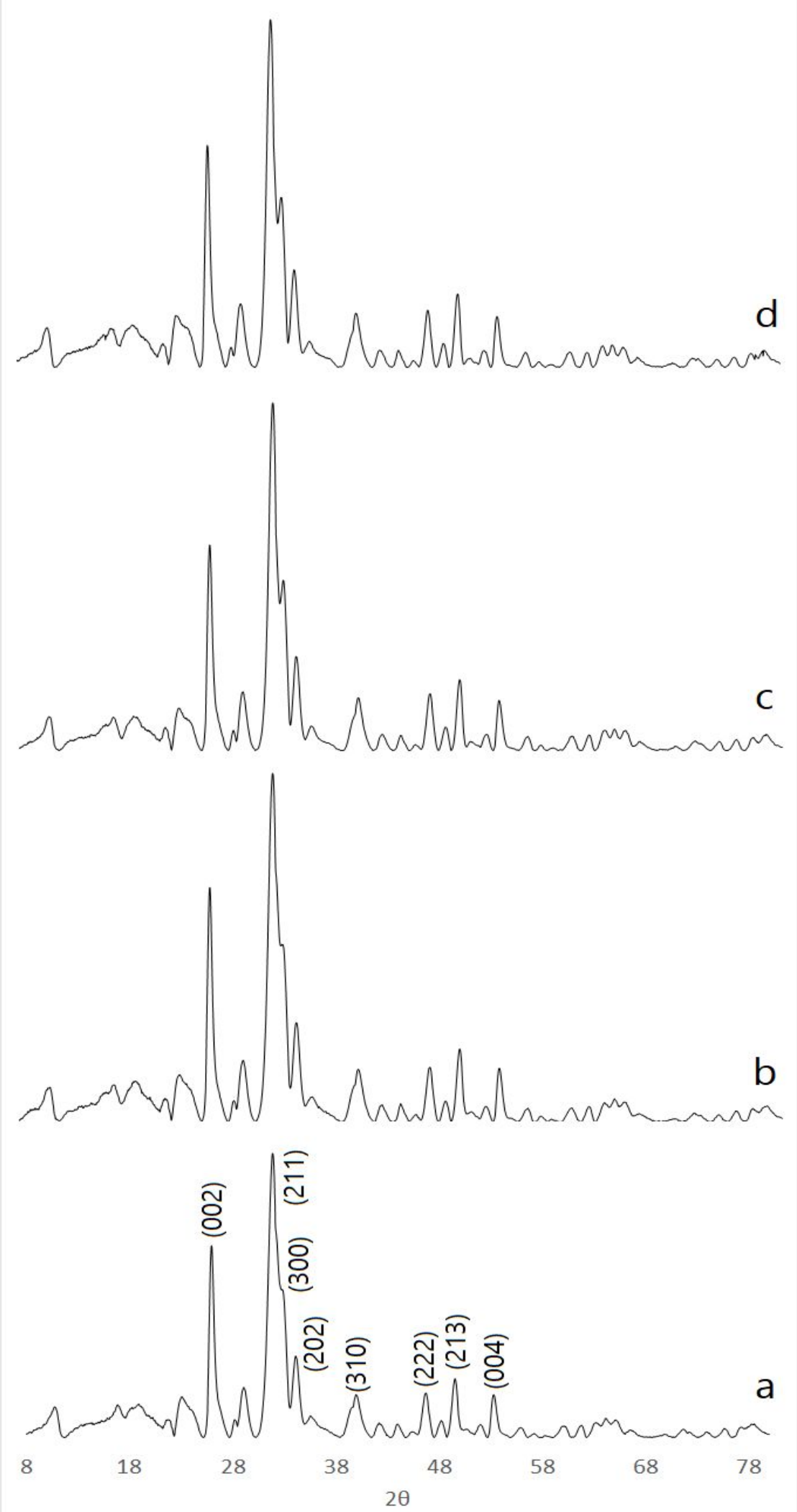

Figure S13. PXRD of (a) HAP, (b) $0.2 \% A u_{19} / \mathrm{HAP}_{-} \mathrm{H}_{2}$, (c) $0.2 \% A u_{20} / \mathrm{HAP}-\mathrm{H}_{2}$, (d) $0.2 \%$ $\mathrm{Au}_{22} / \mathrm{HAP}-\mathrm{H}_{2}$ 


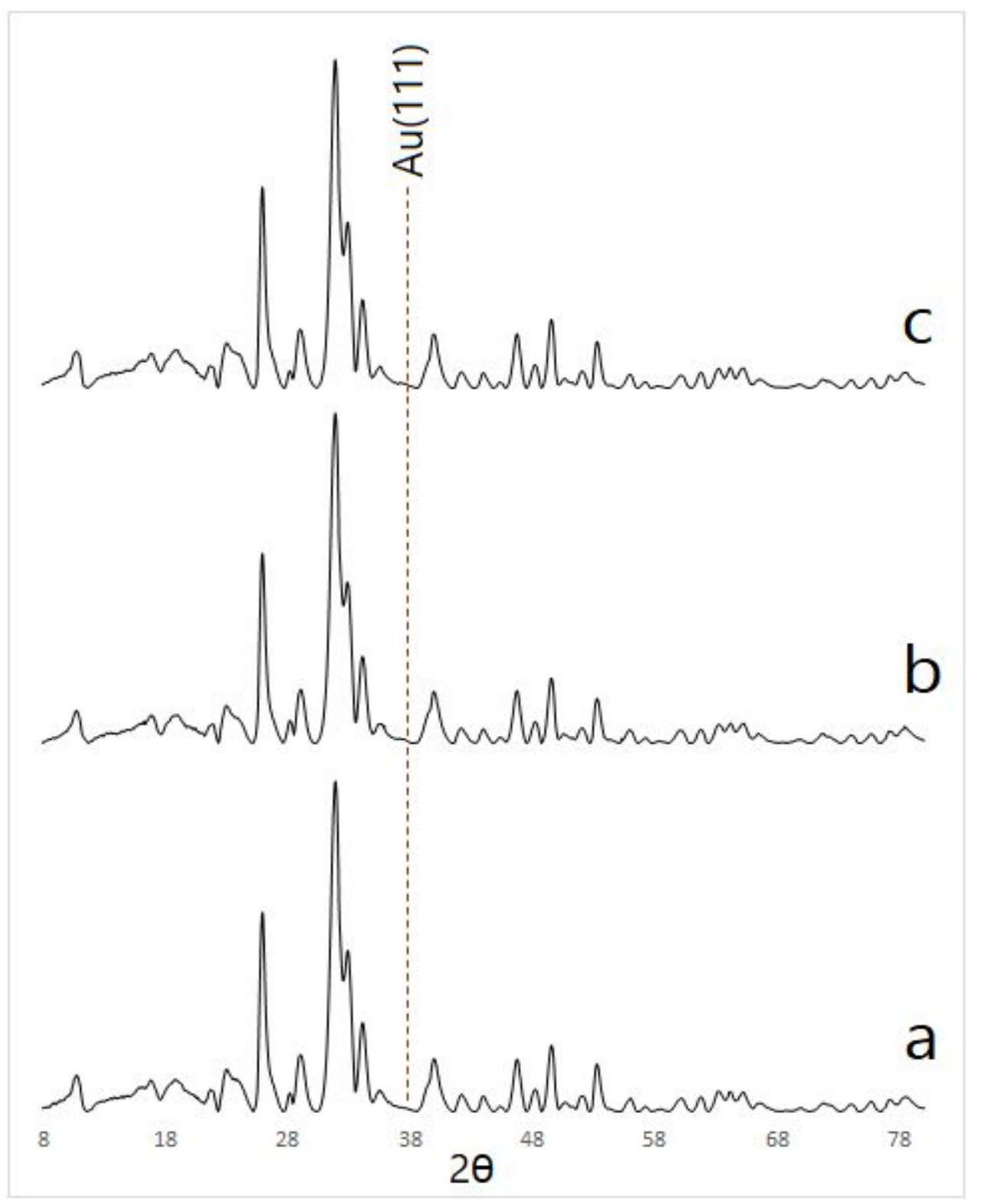

Figure S14. PXRD of (a) $1.0 \% \mathrm{Au}_{1} / \mathrm{HAP}-\mathrm{H}_{2}$, (b) $1.0 \% \mathrm{Au}_{20} / \mathrm{HAP}-\mathrm{H}_{2}$, (c) $1.0 \% \mathrm{Au}_{22} / \mathrm{HAP}-$ H2 


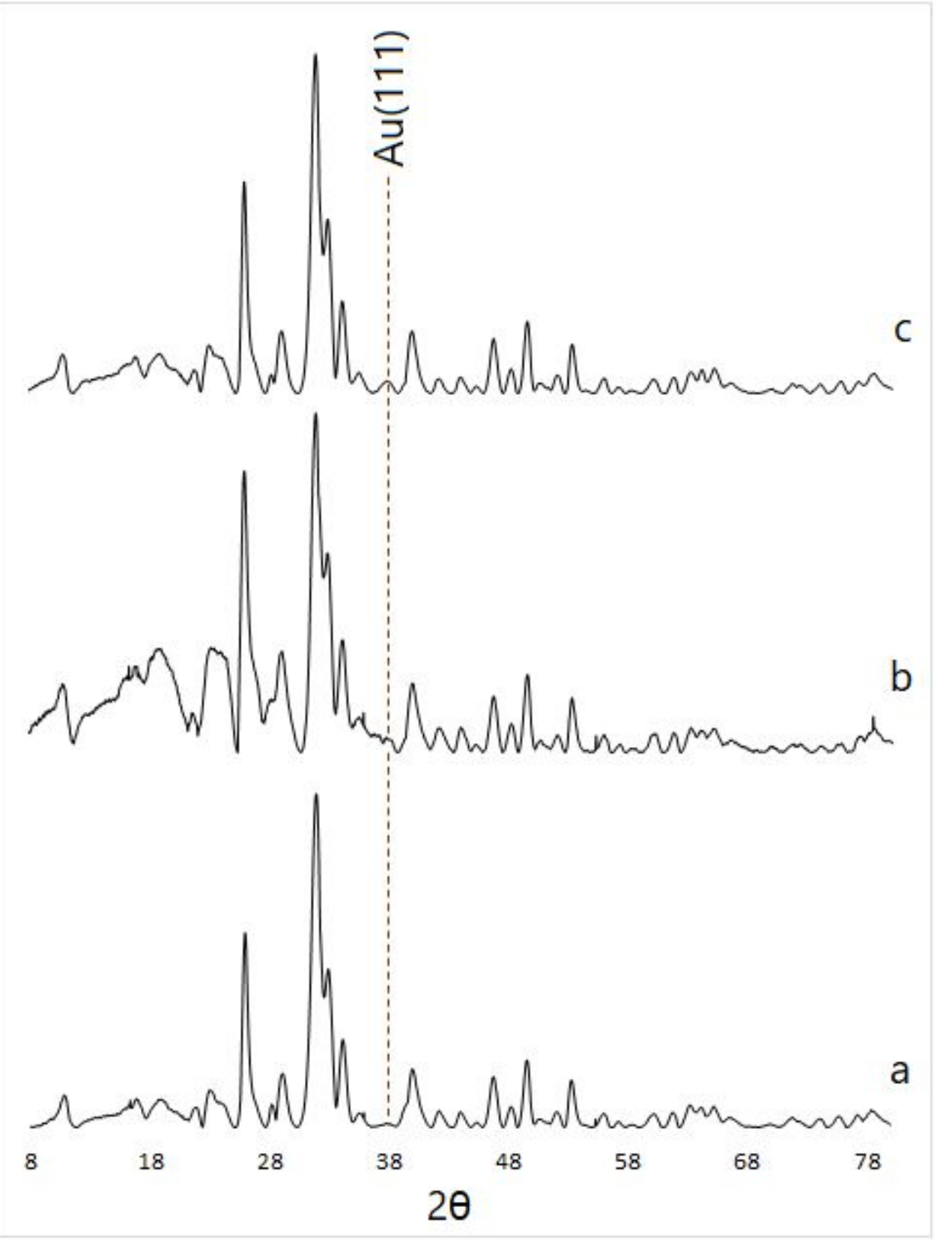

Figure S15. PXRD of (a) 2.5\% Au ${ }_{19} / \mathrm{HAP}_{-} \mathrm{H}_{2}$, (b) $2.5 \% \mathrm{Au}_{20} / \mathrm{HAP}-\mathrm{H}_{2}$, (c) $2.5 \% \mathrm{Au}_{22} / \mathrm{HAP}-\mathrm{H}_{2}$ 


\subsection{UV-Vis DR spectra of selected catalyst materials after catalytic tests}

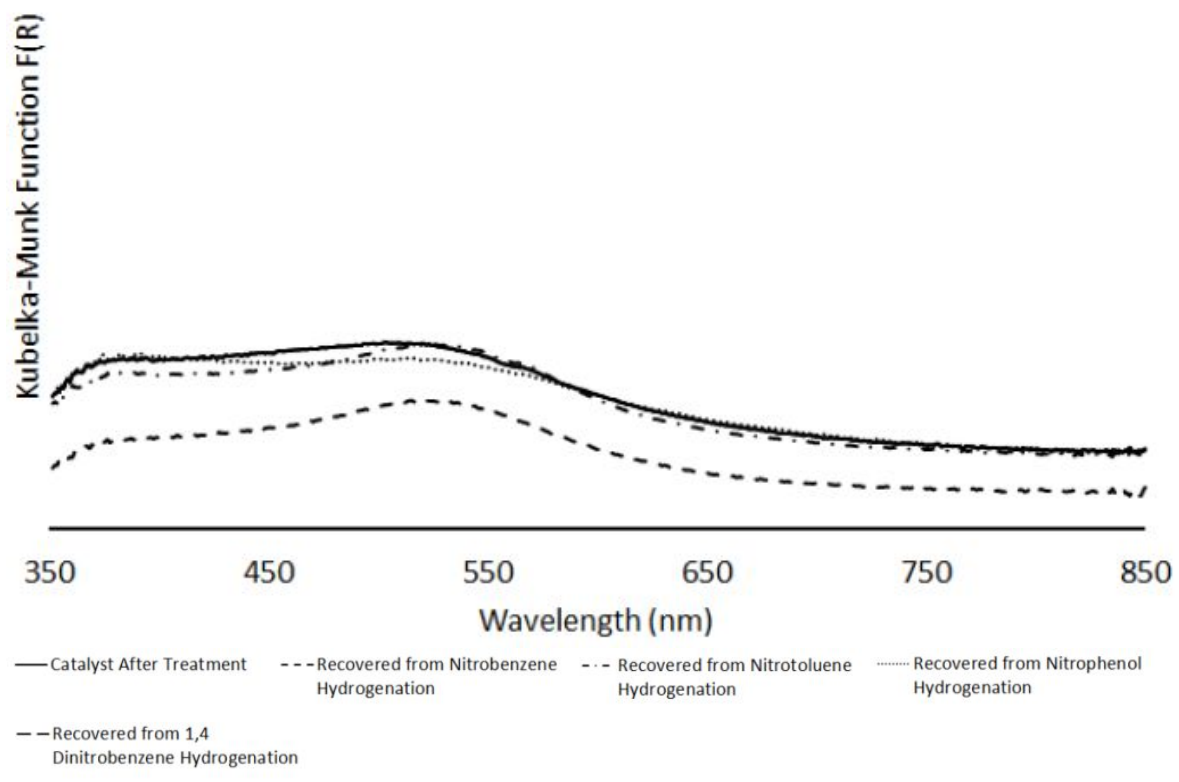

Figure S16. UV-Vis DR Spectra of $0.2 \% \mathrm{Au}_{19} / \mathrm{HAP}$ after recovery from catalytic hydrogenation reactions.

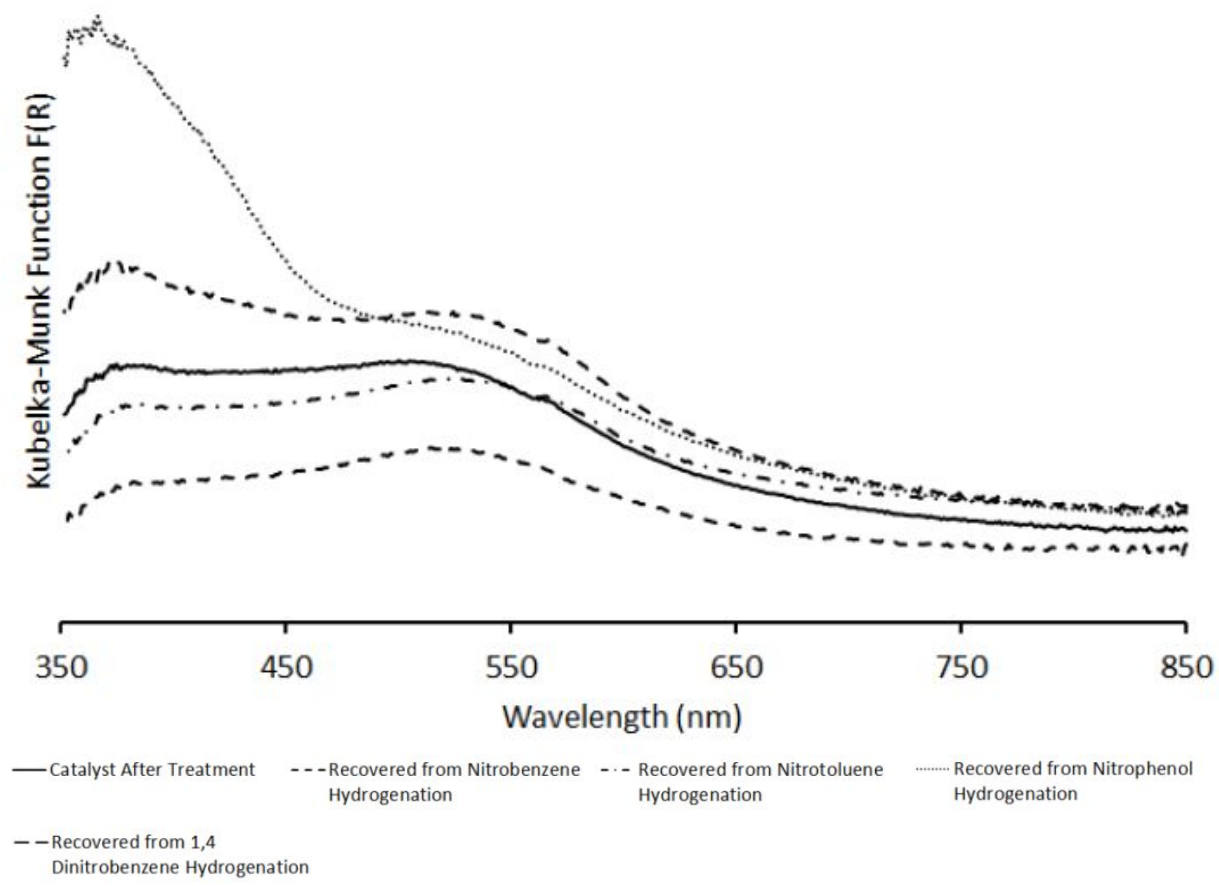

Figure S17. UV-Vis DR Spectra of $0.2 \% A u_{20} / H A P$ after recovery from catalytic hydrogenation reactions. 


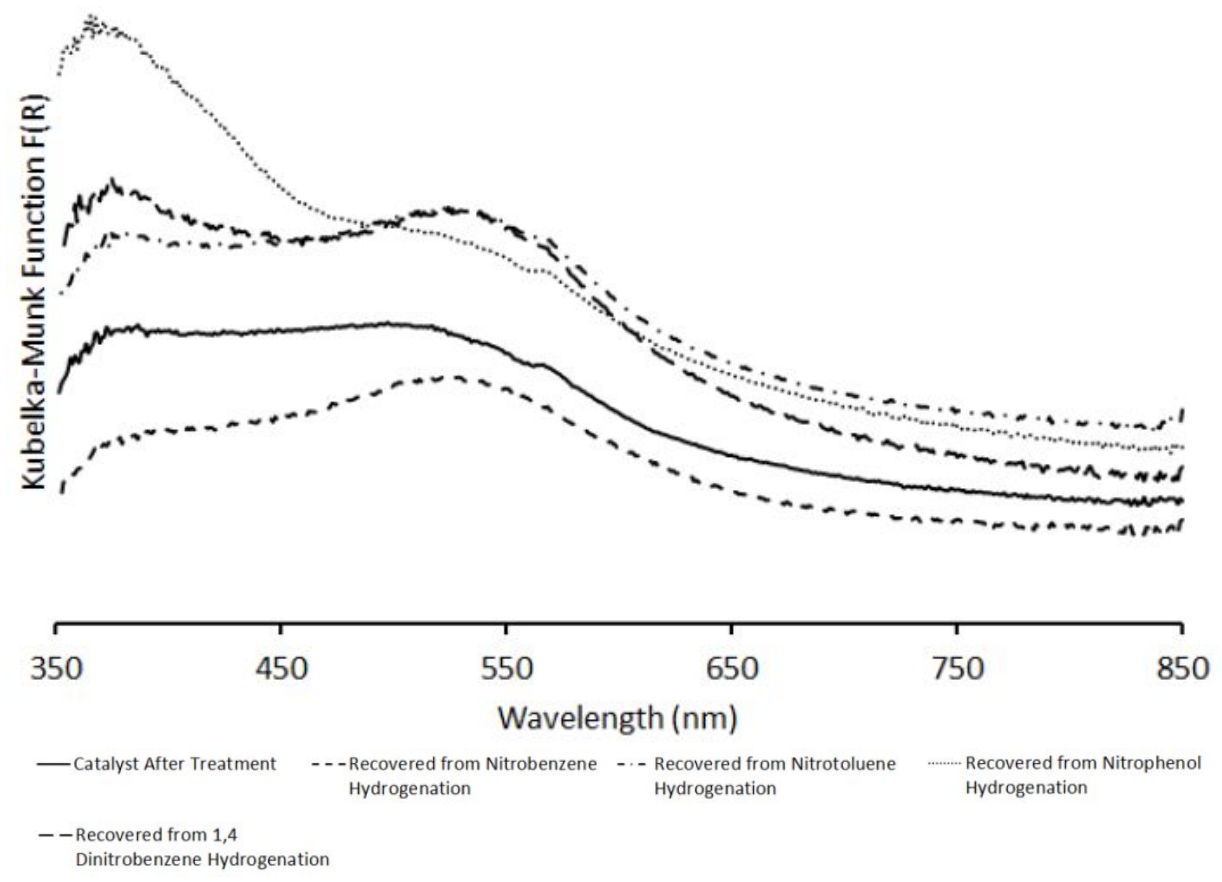

Figure S18. UV-Vis DR Spectra of $0.2 \% \mathrm{Au}_{22} / \mathrm{HAP}$ after recovery from catalytic hydrogenation reactions. 